\title{
Searches for right-handed neutrinos at accelerators
}

\author{
Jan Hajer* \\ Centre for Cosmology, Particle Physics and Phenomenology - CP3 \\ Institut de Recherche en Mathématique et Physique - IRMP \\ Université catholique de Louvain - UCLouvain \\ E-mail: jan.hajer@uclouvain.be
}

Extensions to the SM featuring a low-scale seesaw can be used to to explain the observation of neutrino oscillations, baryogenesis and dark matter. I present the potential to search for right-handed neutrinos using current experiments. I compare the reach of the main detectors at the LHC when a displaced vertex signature in proton collisions is used. Additionally, I show the potential to improve on that using heavy ion collisions. Finally, I present the reach of the fixed target experiment NA62.

European Physical Society Conference on High Energy Physics - EPS-HEP2019 10-17 July, 2019

Ghent, Belgium

${ }^{*}$ Speaker. 


\section{Introduction}

A well motivated minimal extension to the Standard Model (SM) consists of the addition of three right-handed neutrinos via [3]

$$
\mathcal{L}_{\nu_{R}}=-y_{a i} \bar{\ell}_{a} \varepsilon \phi \nu_{R i}-\frac{1}{2} \overline{\nu_{R i}^{c}} M_{i j} \nu_{R j}+\text { h.c. }
$$

where $M_{i j}$ is the Majorana mass. The SM on its own is invariant under a global $B-L$ symmetry, requiring $M_{i j}$ to be small minimizes its breaking. After electroweak symmetry breaking the Yukawa coupling $y_{a i}$ generates a Dirac mass $m_{a i}=v y_{a i}$. Subsequently, the seesaw mechanism $m_{\nu}=-m_{a i} M_{i j}^{-1} m_{b j}^{T}=-\theta_{a i} M_{i j} \theta_{b j}^{T}$, where $\theta_{a i}=m_{a j} M_{i j}^{-1}$ produces tiny masses for the left handed neutrinos and results in a small mixing into mass eigenstates $\nu \simeq U_{\nu}^{\dagger}\left(\nu_{L}-\theta \nu_{R}^{c}\right)$ and $N \simeq \nu_{R}+\theta^{T} \nu_{L}^{c}$. These heavy neutrinos $N$ couple to the bosons of the SM via

$$
\mathcal{L} \supset-\frac{m_{W}}{v} \bar{N} \theta_{a}^{*} \gamma^{\mu} e_{L a} W_{\mu}^{+}-\frac{m_{Z}}{\sqrt{2} v} \bar{N} \theta_{a}^{*} \gamma^{\mu} \nu_{L a} Z_{\mu}-\frac{M}{v} \theta_{a} h \bar{\nu}_{L}{ }_{\alpha} N+\text { h.c. }
$$

The resulting lifetime for light right-handed neutrinos is depicted in Figure 1, while the branching fractions for even lighter right-handed neutrinos into leptons and mesons is depicted in Figure 2. The $\nu$ MSM realizes this low scale type I seesaw and is able to explain not only the neutrino masses, but also neutrino oscillation data, baryogenesis via leptogenesis and has a dark matter candidate [4]. In the following we use the abbreviation $U^{2}=\sum_{a} U_{a}^{2}$, where $U_{a}^{2}=\sum_{i} U_{a i}^{2}$ and $U_{a i}^{2}=\left|\theta_{a i}\right|^{2}$. The ratio $U_{a}^{2} / U_{2}$ is independent of other heavy neutrino parameter and can be calculated from neutrino oscillation data. The resulting probability contours for two active flavours are given in Figure 3.

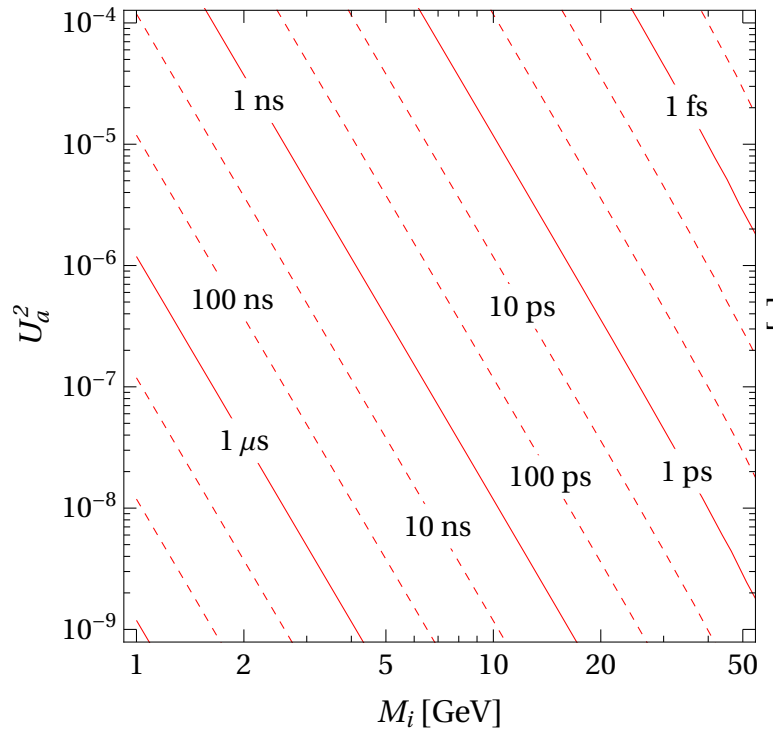

(a) Lifetime

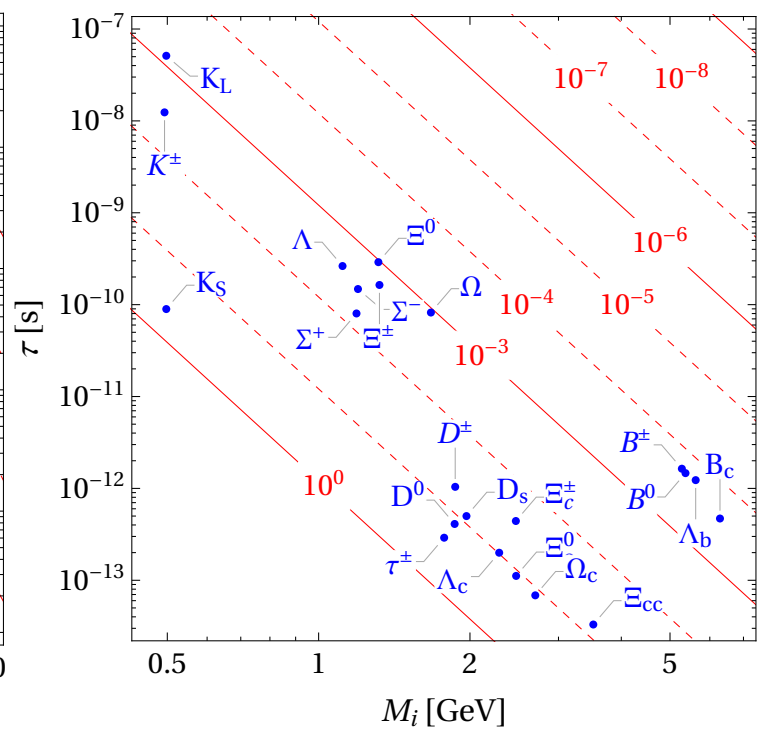

(b) SM particles vs. coupling strength $U^{2}$

Figure 1: Lifetime of right-handed neutrinos calculated with MadGraph5_aMC@NLO. For $M \gg 5 \mathrm{GeV}$ the decay width can be estimated by $\Gamma_{N} \simeq 11.9 \times \frac{G_{F}^{2}}{96 \pi^{3}} U_{a}^{2} M^{5}$. Figure taken from [1]. 


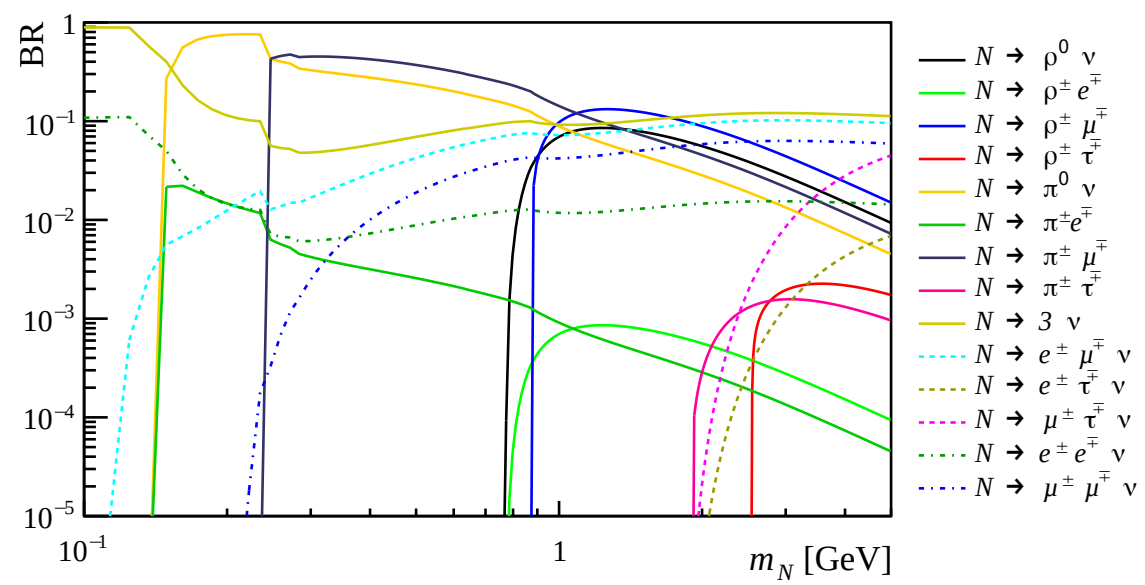

Figure 2: Branching Fractions for light right-handed neutrinos with $U_{i e}^{2}: U_{i \mu}^{2}: U_{i \tau}^{2}=1: 160$ : 27.8. Figure taken from [2].

\section{NA62}

NA62 is a fixed target experiment in the North Area of CERN using the SPS with the goal to measure the very rare kaon decay $K^{+} \rightarrow \pi^{+} \nu \bar{\nu}$ and extract a $10 \%$ measurement of the CKM parameter $\left|V_{t d}\right|$. NA62 can also be used to search for hidden new physics such as a heavy neutrinos as depicted in Figure 4. In its target mode only $K^{+}$induced processes are relevant and in its dump mode $D$ - and $B$-meson induced processes become important. Using a toy Monte Carlo we have simulated heavy neutrino production in $10^{18}$ proton on

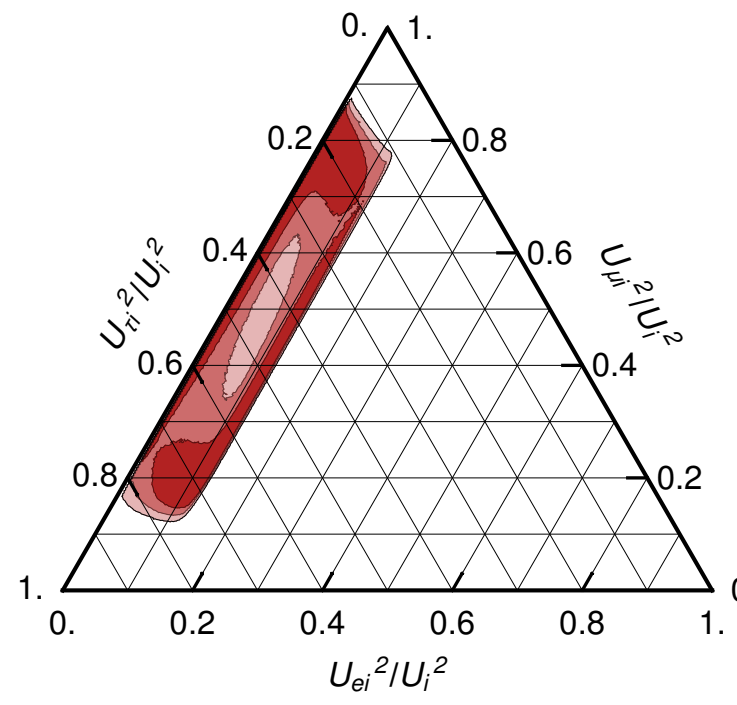

(a) Normal Ordering

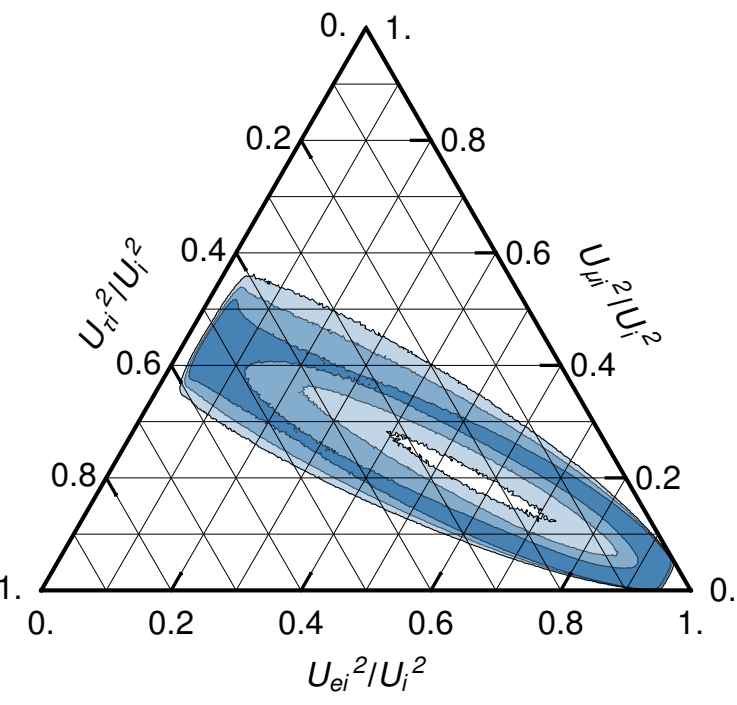

(b) Inverted Ordering

Figure 3: Probability contours for $U_{a}^{2} / U_{2}$ in the case of two active flavours. The coloured areas are consistent with neutrino oscillation data at 1,2 , and $3 \sigma$ and the unknown Majorana phase correspond to the circular structure. Figure taken from [2]. 


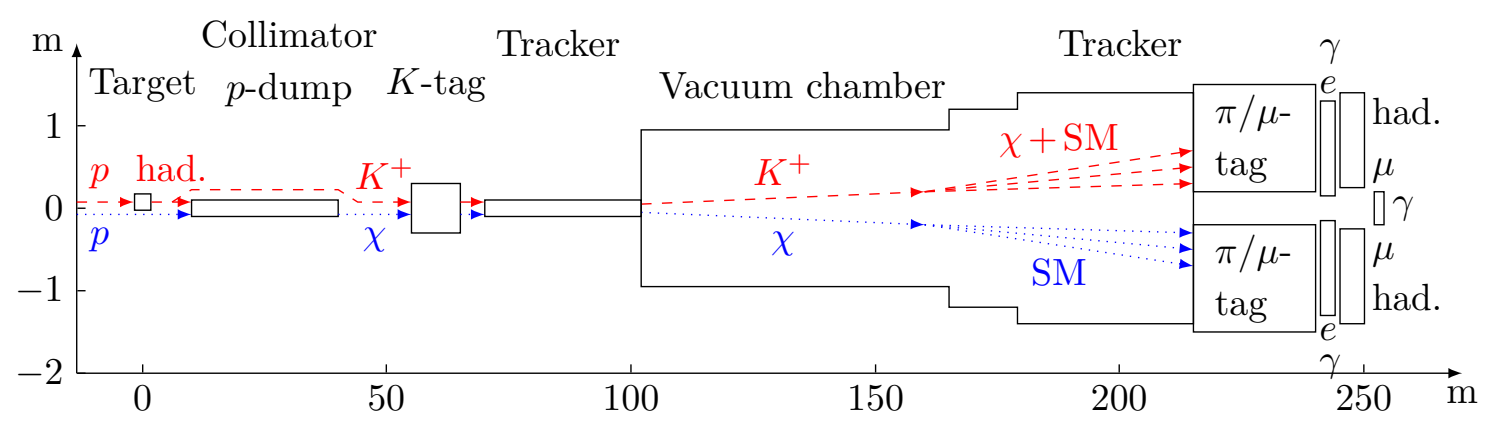

Figure 4: The NA62 experiment. The target mode is depicted in dashed red, the dump mode is shown in dotted blue, and $\chi$ indicates hidden new physics.

target (POT) in the dump mode which corresponds to 80 days of data taking planned for Run 3 (2021-2023). The heavy neutrinos are produced via $2 \times 10^{15} \mathrm{D}$ - and $10^{11} \mathrm{~B}$-mesons

$$
n_{N} \simeq 2 N_{\mathrm{POT}}\left(\chi_{c} f_{D} \mathrm{BR}(D \rightarrow X N)+\chi_{b} f_{B} \mathrm{BR}(B \rightarrow X N)\right),
$$

where $\chi$ is the production cross section and $f$ is the production fractions of mesons. The number of reconstructed events is

$$
N_{\mathrm{obs}}=n_{N} \sum_{f, f^{\prime}=e, \mu, \tau, \pi, K} \operatorname{BR}\left(N_{i} \rightarrow f^{+} f^{\prime-} X\right) \mathcal{A}_{i}\left(f^{+} f^{\prime-} X, M_{i}, U_{e, \mu, \tau}^{2}\right) \varepsilon\left(f^{+} f^{\prime-} X, M_{i}\right)
$$

where $\mathcal{A}_{I}$ is the geometrical acceptance and $\varepsilon$ are the trigger, reconstruction, and selection efficiencies which are assumed to be $100 \%$. Additionally we assume that all backgrounds can be neglected. The dominant decay modes are (cf. Figure 2) $N_{i} \rightarrow$ $3 \nu, \pi^{0} \nu, \pi^{ \pm} \ell^{\mp}, \rho^{0} \nu, \rho^{ \pm} l, \ell^{+} \ell^{-} \nu, \ldots$ and we assume that the detector is able to reconstruct all final states with two charged tracks. The results are presented in Figure 5.

\section{LHC}

We propose to search for right-handed neutrinos using displaced vertices in the LHC [1, 5]. Using the processes shown in Figure 6 one can trigger on the first lepton and search for a secondary vertex. As the muon chamber reaches farther than the tracker it can be advantageous to search for long lived particles using only the muon chambers $[1,6-8]$. For the displaced vertex reconstruction we require at least two tracks with an invariant mass of $5 \mathrm{GeV}$ in order to suppress nuclear interactions backgrounds. The particles must transverse at least half of the tracker or the complete muon chamber. The results of our study are presented in Figure 7, they can be described using a simplified model $N_{d} \sim L_{\text {int }} \sigma_{\nu} U^{2}\left(e^{-l_{0} / \lambda_{N}}-e^{-l_{1} / \lambda_{N}}\right) f_{\text {cut }}$, where $l_{0}$ is the minimal displacement, $l_{1}$ is the detector length, $\lambda_{N}=\frac{\beta \gamma}{\Gamma_{N}}$ is the decay length, and $f_{\text {cut }}$ are all efficiencies.

\section{Heavy Ion Collisions}

Finally we also propose to search for long lived particles such as light right-handed neutrinos in heavy ion collisions [9-11]. The advantages of using heavy ion collisions are 


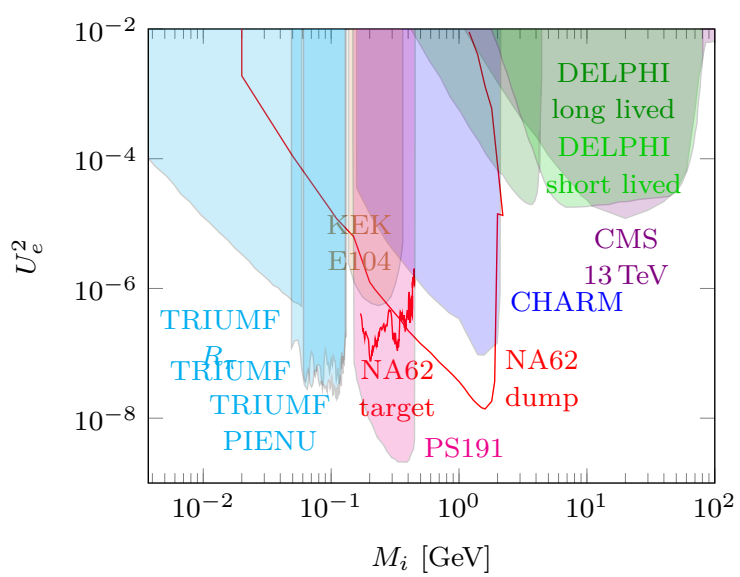

(a) pure $U_{e}^{2}$

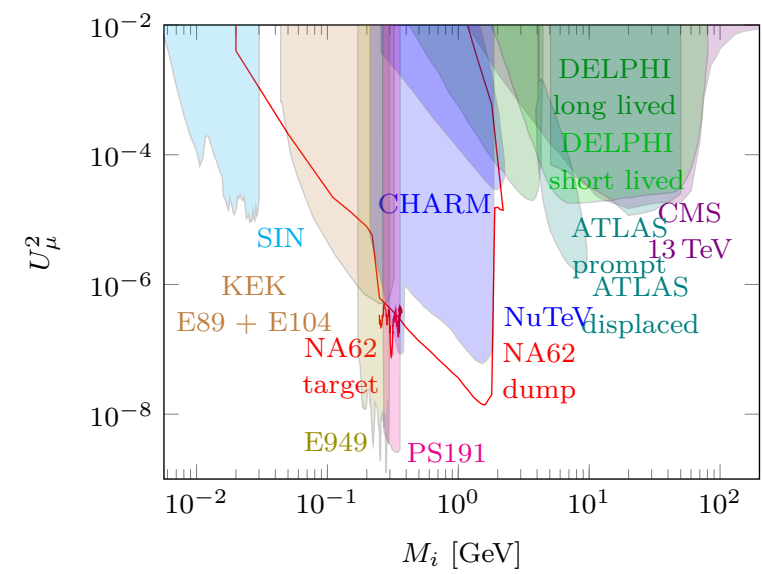

(b) pure $U_{\mu}^{2}$

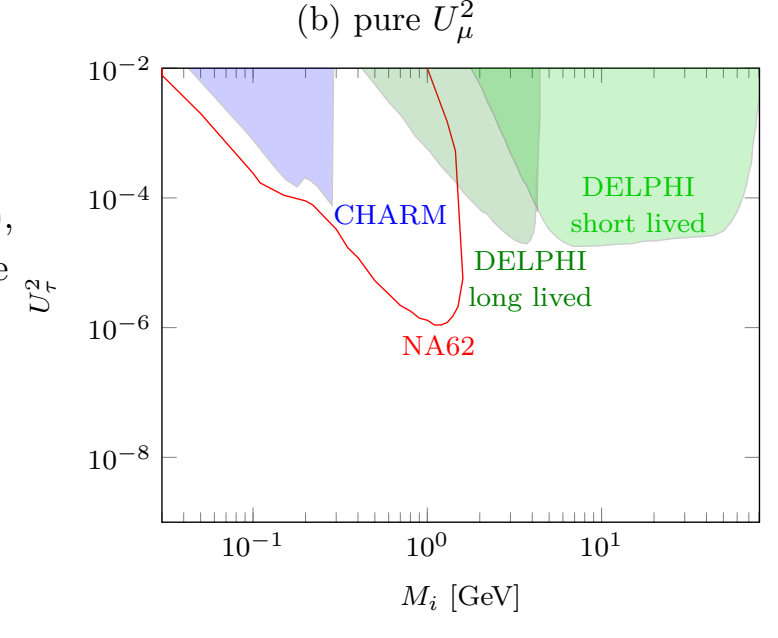

(c) pure $U_{\tau}^{2}$

Figure 5: Exclusions reach at NA62 (red lines), compared to previous experiments. Figure taken from [2].

the absence of pile-up, which leads to a single primary vertex, as well as the large nucleon multiplicity e.g. $A(\mathrm{~Pb})=208, Z(\mathrm{~Pb})=82$, which leads to a large number of parton level interactions per collision as it scales with $A$ e.g. $\frac{\sigma_{\mathrm{PbPb}}}{\sigma_{p p}} \propto A^{2}=43 \times 10^{3}$. The main drawbacks are the huge number of tracks near the interaction point, which makes the search for prompt new physics extremely challenging and the small collision energy per nucleon e.g. $\sqrt{s_{N N}}=5.02 \mathrm{TeV}$ for $\mathrm{Pb}$, which is mostly problematic for heavy new physics. Additionally, the LHC has allocated much less time to heavy ions runs than to protons runs. But

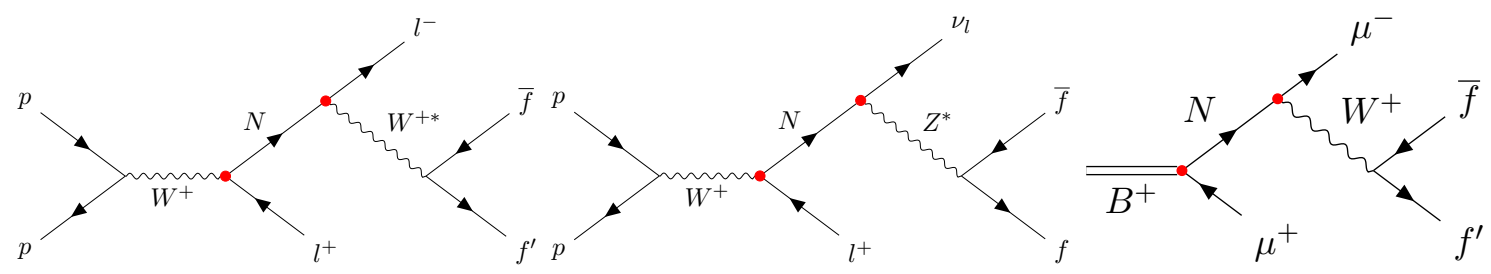

(a) $W$-decay

(b) Z-decay

(c) $B$-production

Figure 6: Displaced signatures at the LHC. 


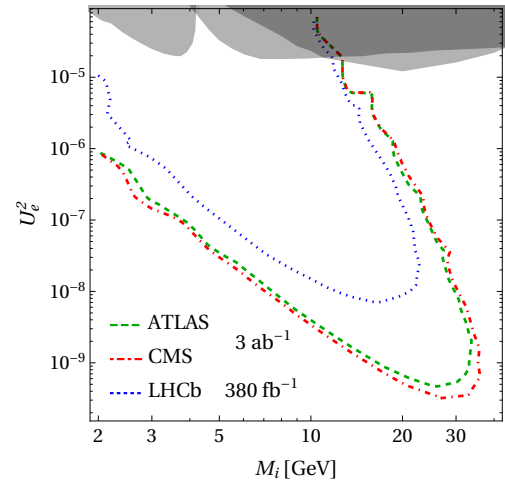

(a) pure $U_{e}^{2}$

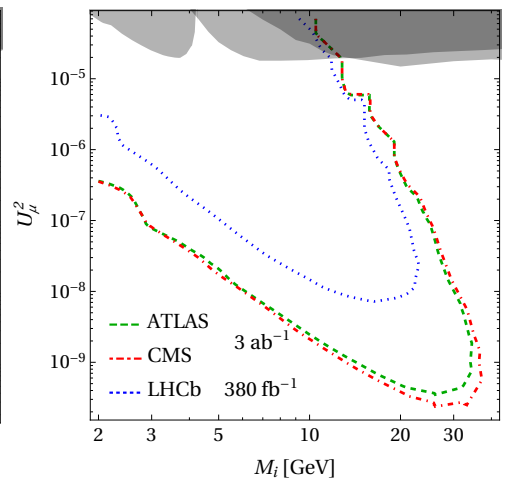

(b) pure $U_{\mu}^{2}$

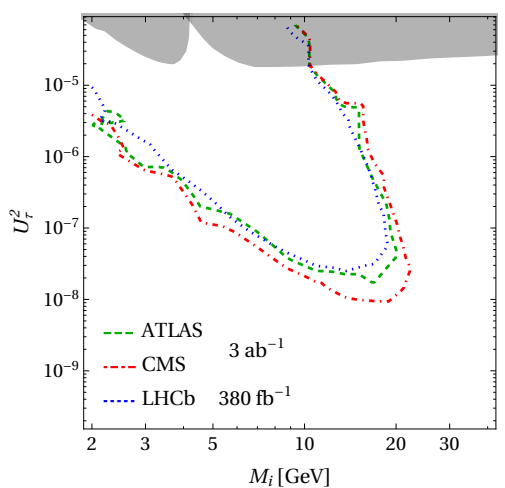

(c) pure $U_{\tau}^{2}$

Figure 7: Maximal exclusion reach at the HL-LHC. Figure taken from [1].

most importantly the instantaneous luminosity is lower for heavier ions. This is caused by additional electromagnetic contributions to the cross-section

Bound-Free Pair Production (BFPP): ${ }^{208} \mathrm{~Pb}^{82+}+{ }^{208} \mathrm{~Pb}^{82+} \stackrel{\gamma}{\rightarrow}{ }^{208} \mathrm{~Pb}^{82+}+{ }^{208} \mathrm{~Pb}^{81+}+e^{+}$ Electromagnetic Dissociation (EMD): ${ }^{208} \mathrm{~Pb}^{82+}+{ }^{208} \mathrm{~Pb}^{82+} \stackrel{\gamma}{\rightarrow}{ }^{208} \mathrm{~Pb}^{82+}+{ }^{207} \mathrm{~Pb}^{82+}+n$

This results in faster beam decay and the production of secondary beams consisting of ions with different charge/mass ratio, which can accidentally quench the magnets [12]. This can be compensated by lowering the triggers which is allowed by the low luminosity and by using lighter ions which allows to increase luminosity again. Our results are presented in Figure 8.

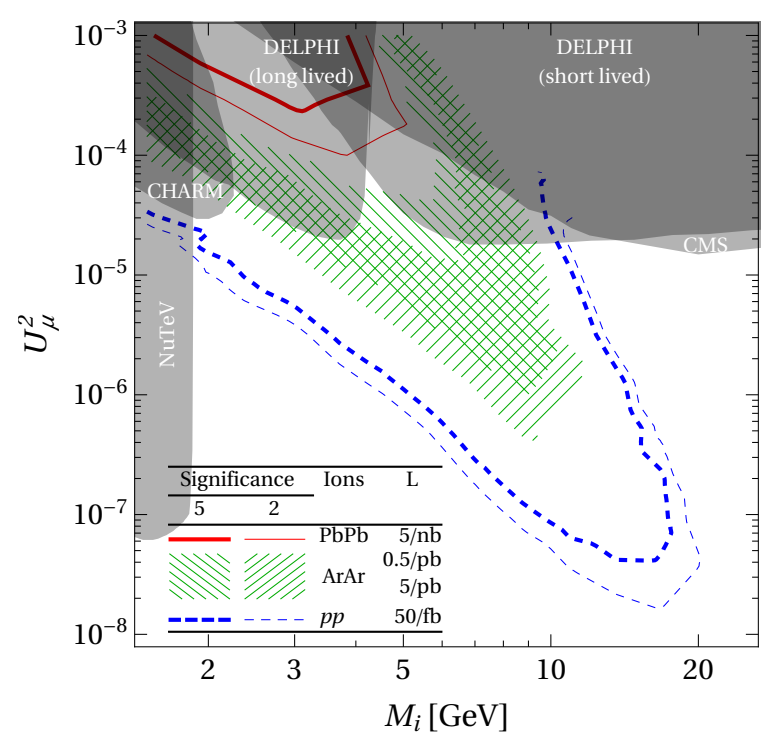

(a) Full simulation of $W$ production

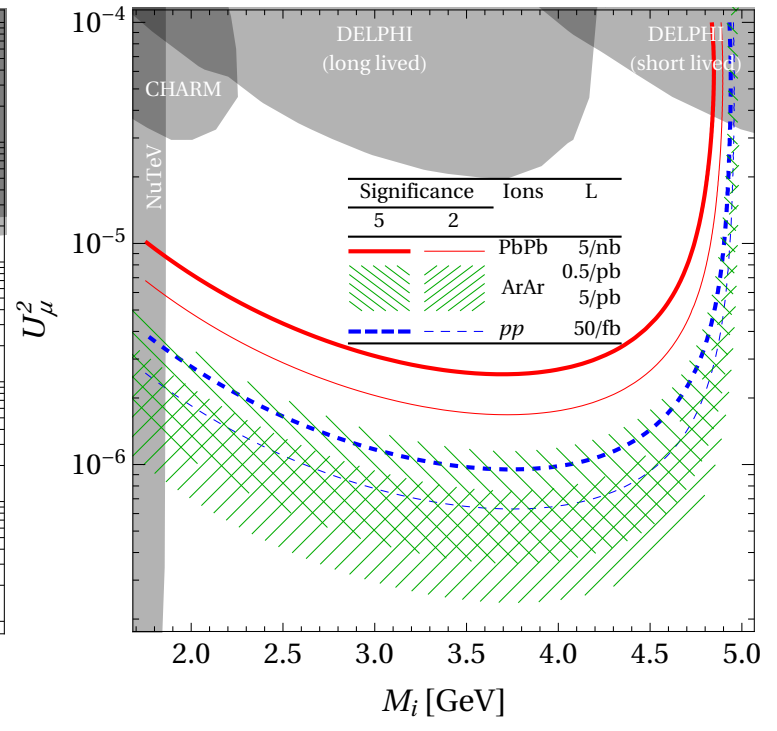

(b) Simplified simulation of $B$ production

Figure 8: Exclusion reach in heavy ion collisions empowered by a low trigger threshold of $p_{T}>3 \mathrm{GeV}$ for heavy ion collisions. Figure taken from [9]. 


\section{Conclusion}

A minimal extension to the SM featuring long lived particles is given by right-handed neutrinos. NA62 is, at the moment, the leading experiment capable of searching for righthanded neutrinos with masses between the $K$ - and $D$-meson mass. Additionally, displaced vertices are a promising signature to detect right-handed neutrinos at the LHC and, finally, heavy ion collisions can also be exploited to search for right-handed neutrinos.

\section{References}

[1] M. Drewes and J. Hajer. "Heavy Neutrinos in displaced vertex searches at the LHC and HL-LHC" (2019). arXiv: 1903.06100 [hep-ph]. №: CP3-19-11.

[2] M. Drewes et al. "NA62 sensitivity to heavy neutral leptons in the low scale seesaw model". JHEP 07 (2018), p. 105. DOI: 10.1007/JHEP07 (2018) 105. arXiv: 1801.04207 [hep-ph].

[3] P. Minkowski. " $\mu \rightarrow e \gamma$ at a Rate of One Out of $10^{9}$ Muon Decays?" Phys. Lett. 67B (1977), pp. 421-428. DOI: 10.1016/0370-2693 (77) 90435-X. №: PRINT-77-0182 (BERN).

[4] T. Asaka and M. Shaposhnikov. "The $\nu$ MSM, dark matter and baryon asymmetry of the universe". Phys. Lett. B620 (2005), pp. 17-26. DOI: 10.1016/j . physletb. 2005. 06.020. arXiv: hep-ph/0505013 [hep-ph].

[5] J. Alimena et al. "Searching for long-lived particles beyond the Standard Model at the Large Hadron Collider" (2019). arXiv: 1903.04497 [hep-ex] .

[6] S. Bobrovskyi et al. "Quasi-stable neutralinos at the LHC". JHEP 09 (2011), p. 119. DOI: 10.1007/JHEP09 (2011) 119. arXiv: 1107.0926 [hep-ph]. №: DESY-11-077.

[7] S. Bobrovskyi, J. Hajer, and S. Rydbeck. "Long-lived higgsinos as probes of gravitino dark matter at the LHC". JHEP 02 (2013), p. 133. DOI: 10.1007/JHEP02 (2013) 133. arXiv: 1211.5584 [hep-ph]. №: DESY-12-175.

[8] CMS. "Search for long-lived particles that decay into final states containing two muons, reconstructed using only the CMS muon chambers" (2015). №: CMS-PASEXO-14-012.

[9] M. Drewes et al. "A Heavy Metal Path to New Physics" (2018). arXiv: 1810.09400 [hep-ph]. №: CP3-18-60.

[10] M. Drewes et al. "Long Lived Particles Searches in Heavy Ion Collisions at the LHC" (2019). arXiv: 1905.09828 [hep-ph]. №: CP3-19-26.

[11] R. Bruce et al. "New physics searches with heavy-ion collisions at the LHC" (2018). arXiv: 1812.07688 [hep-ph].

[12] Z. Citron et al. "Future physics opportunities for high-density QCD at the LHC with heavy-ion and proton beams". 2018. arXiv: 1812.06772 [hep-ph]. №: CERN-LPCC2018-07. 